\title{
Spatio-spectral structures in high harmonic generation driven by tightly focused high repetition rate lasers
}

\author{
Aura Inés Gonzalez, ${ }^{1,2,3, *}$ GaËtan Jargot, ${ }^{1,4}$ Philippe Rigaud, ${ }^{1}$ Loïc Lavenu, ${ }^{1,5}$ Florent Guichard, ${ }^{5}$ \\ Antoine Comby, ${ }^{6}$ Thierry Auguste, ${ }^{2}$ Olivier Sublemontier, ${ }^{7}$ Michel Bougeard, ${ }^{2}$ Yoann Zaouter, ${ }^{5}$ \\ Patrick Georges, ${ }^{1}$ Marc Hanna, ${ }^{1}$ and Thierry Ruchon ${ }^{2}$ \\ 'Laboratoire Charles Fabry, Institut d'Optique Graduate School, CNRS, Université Paris Saclay, 2 Avenue Augustin Fresnel, \\ 91127 Palaiseau, France \\ ${ }^{2}$ LIDYL, CEA, CNRS, Université Paris-Saclay, CEA Saclay 91191 Gif-sur-Yvette, France \\ ${ }^{3}$ Amplitude Technologies, 2 rue du Bois Chaland, 91090 Lisses, France \\ ${ }^{4}$ Fastlite, 1900 route des Crêtes, 06560 Valbonne, Sophia Antipolis, France \\ ${ }^{5}$ Amplitude Systemes, 11 Avenue de Canteranne, Cité de la Photonique, 33600 Pessac, France \\ ${ }^{6}$ Université de Bordeaux-CNRS-CEA, CELIA, UMR5107, F33405 Talence, France \\ ${ }^{7}$ NIMBE, CEA, CNRS, Université Paris-Saclay, CEA Saclay 91191 Gif-sur-Yvette, France \\ *Corresponding author: aura.gonzalez@amplitude-laser.com
}

Received 30 October 2017; revised 25 January 2018; accepted 25 January 2018; posted 25 January 2018 (Doc. ID 310222$)$; published 27 February 2018

\begin{abstract}
We investigate the spatio-spectral properties of extreme ultraviolet (XUV) high harmonic radiation driven by high repetition rate femtosecond laser systems. In the spatio-spectral domain, ring-shaped structures at each harmonic order associated with long-trajectory electrons are found to form arrow-shaped structures at the cutoff. These structures are observed with two different laser systems: an optical parametric chirped-pulse amplifier system at a central wavelength of $1.55 \mu \mathrm{m}$ and $125 \mathrm{kHz}$ repetition rate, and a temporally compressed femtosecond ytterbium fiber amplifier at $1.03 \mu \mathrm{m}$ wavelength and $100 \mathrm{kHz}$ repetition rate. As recently pointed out, the observed structures are well explained by considering the space-time atomic dipole-induced phase for short and long electron trajectories in the generation plane. The tighter focusing geometry and longer wavelength associated with these emerging driving laser systems increase the ring-like divergence and spectral broadening for high harmonics. Cutoff energies and photon fluxes obtained in argon and neon are also reported. Overall, these results shed new light on the properties of XUV radiation driven by these recently developed high average power laser systems, paving the way to high photon-flux XUV beamlines. (๑) 2018 Optical Society of America
\end{abstract}

OCIS codes: (340.7480) X-rays, soft x-rays, extreme ultraviolet (EUV); (320.7110) Ultrafast nonlinear optics; (320.7090) Ultrafast lasers.

https://doi.org/10.1364/JOSAB.35.0000A6

\section{INTRODUCTION}

The process of high harmonic generation (HHG) allows the generation of coherent femtosecond to attosecond extreme ultraviolet (XUV) beams by focusing a high-intensity femtosecond laser in a gas medium $[1,2]$. The associated XUV spectrum is typically composed of odd harmonics of the driving laser of approximately constant amplitude, extending up to the photon energy cutoff [3]. Depending on the experimental parameters, such as driving laser pulse duration and central wavelength, interaction geometry, and ionization potential of the gas, the spatial and temporal/spectral properties of the XUV beams can be vastly different [4]. The HHG process can be described using the classical three-step model [3], where the laser field distorts the Coulomb potential of the atom, allowing tunneling of an electron wave packet from the valence shell. This electron is then freely accelerated by the electric field, and can recombine with the parent ion, releasing the acquired potential and kinetic energy in the form of an attosecond XUV burst. This process is repeated every half-cycle of the driving laser, inducing the odd harmonic structure of the radiated XUV beam. Both the semiclassical and quantum [5] analyses of this picture allows a good understanding of this intricate process, which leads to rich spatial and spectral features of the HHG beam. Since HHG is seen nowadays as a unique way to provide XUV photons, enabling 
numerous applications including ultrafast spectroscopy, nanoscale imaging, and attosecond science [6-8], it is essential to understand these spectral and spatial properties to be able to tailor the XUV beam for specific applications.

The majority of recent HHG experiments have been performed with Ti:Sa laser systems that are able to deliver short $(<30 \mathrm{fs})$ energetic pulses (few millijoules) at repetition rates limited to a few kilohertz, corresponding to average powers about $10 \mathrm{~W}$. However, there is currently considerable technological and research effort toward efficient HHG setups compatible with emerging laser systems [9] that are scalable to higher average powers, and typically operate at much higher repetition rate $(\geq 100 \mathrm{kHz})$ and lower energy per pulse. In particular, ytterbium-doped fiber-based amplifier (YDFA) sources have allowed unprecedented XUV photon fluxes [10-13]. Optical parametric chirped-pulse amplifier (OPCPA) systems add the possibility to operate at longer central wavelengths in order to reach higher cutoff energies, and are also increasingly used to drive HHG [14]. The lower pulse energy delivered by these systems requires a tighter focusing geometry to reach intensities of a few $10^{14} \mathrm{~W} / \mathrm{cm}^{2}$ typically required for HHG. The short-associated Rayleigh range asks for short and dense gas targets. These high repetition rate XUV systems are of great interest for several applications, such as coincidence detection of ionization fragments [15] and photoemission spectroscopy [16].

There has been a large number of reports of the spatiospectral features of HHG, including various physical effects such as multiple quantum paths interference [17-20], Maker fringes related to phase-matching effects [21], and space-time atomic dipole phase [22]. These works have been done with Ti:Sa systems at a central wavelength of $800 \mathrm{~nm}$. In this paper, we investigate spatio-spectral HHG features generated with two high repetition rate laser systems: (i) an OPCPA operating at a wavelength $\lambda=1.55 \mu \mathrm{m}$ and delivering pulses with an energy $E=20 \mu \mathrm{J}$ and duration $\tau=50 \mathrm{fs}$ at a repetition rate of $125 \mathrm{kHz}$, and (ii) a temporally compressed YDFA delivering $\lambda=1.03 \mu \mathrm{m}, \tau=30 \mathrm{fs}, E=150 \mu \mathrm{J}$ pulses at a repetition rate of $100 \mathrm{kHz}$. The experimentally observed structures are compared to theoretical results obtained considering a single longitudinal plane, neglecting phase-matching-related effects. All the observed features are compatible with a temporal and purely transverse spatial dependence of the dipole phase. The effects are more pronounced than in the case of Ti:Sa driven HHG because the dipole phase impact increases with stronger focusing and larger driving wavelengths. In addition to these observations, we provide cutoff energies and photon fluxes obtained with these sources, and conclude by discussing implications for users in terms of possible XUV beamline parameters.

\section{EXPERIMENTAL SETUP FOR HIGH HARMONIC GENERATION}

\section{A. OPCPA System at $\lambda=1.55 \mu \mathrm{m}$}

The OPCPA system is based on a commercial high-energy femtosecond ytterbium-doped fiber amplifier (Amplitude Systemes Tangerine) that delivers $400 \mu \mathrm{J}, 400$ fs pulses at $125 \mathrm{kHz}$ and a central wavelength of $1.03 \mu \mathrm{m}$. A small fraction of the pulse energy is used to generate a supercontinuum, constituting the seed for further amplification at a central wavelength of $1550 \mathrm{~nm}$. This allows robust temporal synchronization between the pump and signal. Stretching and compression is achieved using bulk materials, silicon, and fused silica. Three optical parametric amplifier stages based on periodically poled magnesium-doped lithium niobate are implemented. The collinear interaction geometry results in simultaneously usable signal and idler beams at $\lambda=1.55 \mu \mathrm{m}$ and $\lambda=3.07 \mu \mathrm{m}$, respectively. Only the $\lambda=1.55 \mu \mathrm{m}$ beam is used in this work. More details on this source are available in [23]. The output parameters at $\lambda=1.55 \mu \mathrm{m}$ are as follows: pulse duration $50 \mathrm{fs}$, pulse energy $20 \mu \mathrm{J}$, and repetition rate $125 \mathrm{kHz}$.

\section{B. Temporally Compressed YDFA System at $\lambda=1.03 \mu \mathrm{m}$}

The second source is also based on a commercial high-energy femtosecond ytterbium-doped fiber amplifier (Amplitude Systemes Tangerine), but the beam at $\lambda=1.03 \mu \mathrm{m}$ is directly used to perform HHG. However, since shorter pulse durations allow overall better HHG performance, special care has been taken to counteract gain narrowing, allowing the generation of $150 \mathrm{fs}$ pulses at the output of the laser [24]. These pulses are sent to a temporal compression stage that consists of a xenon-filled capillary inducing self-phase modulation followed by chirped mirrors. This compression stage can be entirely bypassed to drive the HHG with 150 fs pulses, or used with various Xe pressures and number of bounces on the chirped mirrors to provide tunable pulse duration at the output, from $16 \mathrm{fs}$ to $30 \mathrm{fs}$ in this work. More information on this source can be found in [24].

\section{HHG Setup}

The HHG setup is depicted in Fig. 1. It consists of a generation chamber and a detection chamber, connected through a $3 \mathrm{~mm}$

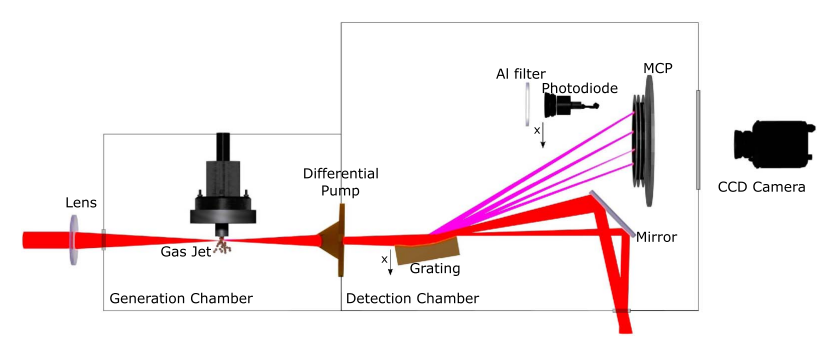

Fig. 1. Experimental setup. The XUV source is composed of two vacuum chambers. The generation chamber has been designed to evacuate the maximum of gas injected at backing pressures up to 12 bars, allowing overall pressures in the chamber of $\sim 10^{-3}$ mbar. The detection chamber hosts an XUV spectrometer with a grating placed on a motorized holder with nanometer precision of the rotation and transversal position. A motorized holder allows insertion of a $200 \mathrm{~nm}$ thick aluminum filter and a calibrated photodiode in the beam path. The spectrum is finally detected on a two-stage MCP in chevron configuration of $77 \mathrm{~mm}$ diameter coupled to a phosphore screen (P43 type). Part of the IR beam is filtered out by a water-cooled skimmer that also acts as a differential pumping between the chambers. The residual IR beam is sent out of the detection chamber with a silver mirror. This part of the IR beam can be used to monitor the IR parameters and beamline alignment. 
diameter skimmer hole that allows propagation of the harmonic beam. It also allows us to establish a differential pressure between the chambers (typically $10^{-3} \mathrm{mbar}$ in the generation chamber and $10^{-5} \mathrm{mbar}$ in the detection chamber). The generating medium is a continuous flow of gas (argon or neon) emitted from a $100 \mu \mathrm{m}$ nozzle backed with a pressure of 3-12 bars. The laser beam is expanded to a diameter of $7 \mathrm{~mm}$ before being focused with a $75 \mathrm{~mm}$ or $150 \mathrm{~mm}$ focal length lens through a $3 \mathrm{~mm}$ thick fused silica window at the output of the gas nozzle. The longitudinal gas jet position with respect to the laser focus is adjustable as an optimization parameter for the experiments; however, its absolute position is not accessible. The HHG beam passes through the skimmer and enters a spectrometer, composed of a 1200 lines per mm Hitachi aberration-corrected grating (001-0266) at grazing incidence and a micro-channel plate (MCP) detector, equipped with a phosphor screen. This screen is imaged onto a CCD camera. This detector is located $50 \mathrm{~cm}$ away from the HHG generation point.

To calibrate the spectrometer in photon energy, a fit is performed considering the grating characteristics and geometry of the setup in terms of angle and distance. This fit is checked using a $200 \mathrm{~nm}$ thick Al filter with a band edge at $75 \mathrm{eV}$ that can be inserted in the XUV beam path. Finally, photon flux measurements are performed using a calibrated XUV photodiode placed downstream the Al filter, after removal of the spectrometer grating. Its current is read by a precision picoammeter and corrected for a small offset due to the remaining infrared light passing the $\mathrm{Al}$ filter. To measure this offset, a differential measurement of the photodiode current with and without generating gas, and in linear or circular polarization, is carried out. The signal is measured as a function of photodiode lateral position with respect to the optical axis, and the XUV signal shows up as a very localized contribution, while the IR background (representing typically $5 \%$ of the signal) is always present. This total photon flux is then used to calibrate the spectra recorded on the CCD camera in terms of photon flux spectral density. Uncertainties on the spectral responses of the photodiode, grating, and $\mathrm{MCP} / \mathrm{phosphor}$ screen result in error bars for the measured photon flux densities of $\pm 30 \%$.

\section{DIPOLE MODEL AND SIMULATIONS}

\section{A. Atomic Phase Model}

The harmonic generation process can be described by a very simple model [22] where the source term for a given harmonic number $q$ and trajectory class (short or long) is written in the spatio-temporal domain at the interaction point as

$$
d_{q}(x, t)=E^{q_{\text {eff }}}(x, t) \exp (-i \alpha I(x, t)),
$$

where $t$ is time, $x$ is the resolved transverse spatial coordinate, $E$ and $I$ are the driver laser field and intensity, and $q_{\text {eff }}$ and $\alpha$ are considered as constant for a fixed harmonic number, type of trajectory, and maximum laser intensity. The $q_{\text {eff }}$ constant describes how the dipole response intensity varies as a function of fundamental field intensity, while $\alpha$ describes the strength of the atomic response-induced phase, which can be shown to be proportional to the driving laser intensity. The value of $\alpha$ (expressed in $10^{-14} \mathrm{rad} \cdot \mathrm{cm}^{2} / \mathrm{W}$ throughout this paper) is related to the time of flight of the electron wave packet in the
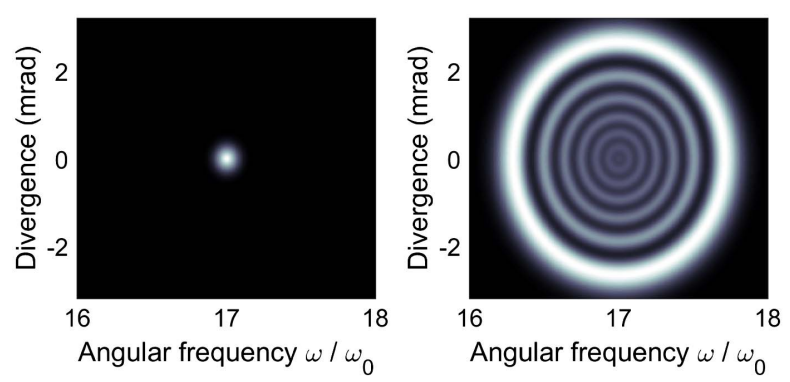

Fig. 2. Intensity in the spatio-spectral domain in the far field calculated using the simple atomic phase model for short trajectories (left, $q_{\text {eff }}=4, \alpha=2$ ) and long trajectories (right, $q_{\text {eff }}=4, \alpha=20$ ).

continuum, and therefore increases with longer driving wavelength with a $\lambda^{3}$ dependence [25]. For short trajectories, this value increases (in absolute value) with harmonic number, while for long trajectories the absolute value of $\alpha$ decreases with harmonic number, merging at the cutoff energy.

To illustrate the consequences of this model, the intensity of the 17 th harmonic order $(26.3 \mathrm{eV})$ in the spatio-spectral plane in the far field is plotted in Fig. 2. The driving laser is considered to deliver $40 \mathrm{fs}$ pulses at $800 \mathrm{~nm}$, focused on a $120 \mu \mathrm{m}$ waist radius at a maximum intensity of $2 \times 10^{14} \mathrm{~W} / \mathrm{cm}^{2}$. The $q_{\text {eff }}$ value is taken to be 4 for both trajectory types, and we have taken $\alpha=2$ for short trajectories and $\alpha=20$ for long trajectories, typical values for argon at $800 \mathrm{~nm}$ [22]. For large values of the dipole-induced phase (long trajectories), the atomic phase term in the near-field temporal domain translates into a ring structure in the far-field spatio-spectral domain. To understand this feature, an interesting analogy with self-phase modulation (SPM) in nonlinear optics can be drawn: in both cases, a phase proportional to the intensity profile is induced. It is well known that for smooth Fourier transform-limited pulses, SPM imparts a spectral broadening that exhibits multiple lobes, which are more prominent on the spectral edges. The overall width of the spectrum and number of lobes increases with the nonlinear phase excursion. The exact same behavior is observed for the atomic phase (in 2D in the case of Fig. 2), with the slight modification that the intensity of the radiating source term is also modified through the value of $q_{\text {eff }}$. Overall, this simple model predicts two contributions to the intensity pattern in the far-field spatio-spectral domain. The short trajectories bring a localized contribution. In contrast, the long trajectories contribute to a more dilute ring pattern, and specific numbers for the divergence and harmonic spectral width depend on the exact values of $q_{\text {eff }}$ and $\alpha$, together with the driving field parameters, such as pulse duration, focal spot size, position, and intensity.

\section{B. Numerical Simulations (SFA Model)}

To obtain more accurate theoretical results, a software package was used to compute the HHG far-field spatio-spectral intensity [26]. This package solves the time-dependent Schrödinger equation under the strong-field approximation (SFA) [5] to compute the nonlinear dipole response to the pulsed driving field at discrete locations in the gas target. Contributions from all the volume regions in the target are then propagated to 

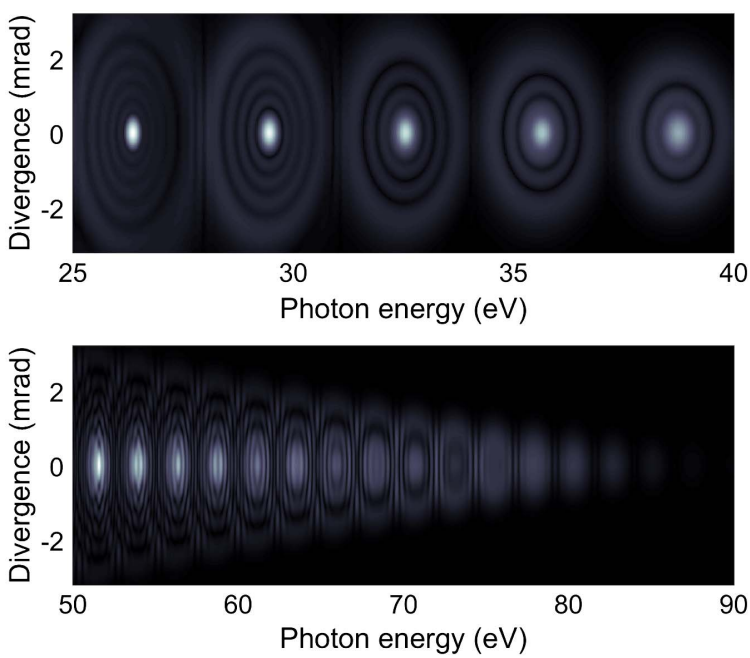

Fig. 3. Intensity in the spatio-spectral domain in the far field calculated using the numerical model based on SFA for central wavelengths of $800 \mathrm{~nm}$ (top), and $1030 \mathrm{~nm}$ (bottom).

include macroscopic effects such as phase matching. Finally, the resulting HHG far field at a specific distance can be obtained. To validate this model, it was first run with a set of parameters used in [22]: generation in a single longitudinal plane of argon with a central wavelength of $800 \mathrm{~nm}$, waist $120 \mu \mathrm{m}$, pulse duration $40 \mathrm{fs}$, and intensity $2 \times 10^{14} \mathrm{~W} / \mathrm{cm}^{2}$. The result is shown in Fig. 3, and is in excellent agreement with the data from [22]. In addition, there is also a remarkable agreement with the simple model described above (the 17th harmonic is the leftmost harmonic in Fig. 3 top).

To get a first illustration of the effect of the central wavelength shift toward longer values, the simulation is reproduced with the same parameters except for the central wavelength, which is changed to $1030 \mathrm{~nm}$. The result is plotted near the energy cutoff in Fig. 3 (bottom). The shift to a longer wavelength results in an extended cutoff energy, and the ring structures associated with neighboring harmonics start to overlap. This is both due to the fact that the value of $\alpha$ increases with wavelength, and to the fact that in a constant spectral zone, the separation of the harmonic orders decreases with the driving wavelength. The overlap between structures induces an interference pattern, whose location is sensitive to the carrierenvelope phase (CEP). They appear as a series of vertical fringes in the spectrum in Fig. 3. This interference will therefore be washed out if observed with a laser that is not CEP-stabilized. Note that since a single transverse plane was considered in these simulations, longitudinal phase matching plays no role in the appearance of the spatio-spectral structures.

These two theoretical tools are used to analyze the experimental data obtained in various conditions, presented hereafter.

\section{EXPERIMENTAL RESULTS}

\section{A. Results Obtained with the YDFA in Argon}

We first describe results obtained using the YDFA laser temporally compressed down to $30 \mathrm{fs}$. For this part, the input pulse $(115 \mu \mathrm{J}$ energy) was focused using a $150 \mathrm{~mm}$ lens in an argon gas jet with a backing pressure of 6.5 bar. The goal of this section is to identify experimentally the origin of the ring structure, and check that our models can predict them with reasonable accuracy. The generating conditions (intensity, position of the gas nozzle, backing pressure) are therefore optimized to observe a clear spatio-spectral pattern. A pinhole is used at the input of the chamber to reduce the input beam size and pulse energy, resulting in a $30 \mu \mathrm{m}$ waist radius at the gas target location and an estimated intensity of $0.7 \times 10^{14} \mathrm{~W} / \mathrm{cm}^{2}$. The spectrometer is aligned to observe photon energies from 20 to $65 \mathrm{eV}$.

Figure 4 shows the experimentally observed spatio-spectral structure in these conditions, along with results from the SFA and atomic phase models. To obtain the spatio-spectral structure with the atomic phase model, the contribution of each harmonic and trajectory is calculated independently using $\alpha$ values obtained from the SFA code, and the contributions' intensities are summed to obtain the final result.

The $\alpha$ values used in this result are plotted in Fig. 5, showing that the longer driving wavelength compared to Ti:Sa systems results in significantly higher transverse spatial phases.

Overall, the agreement between the experimental data and both models is good in terms of divergence observed for each harmonic. In particular, as the photon energy increases, since $\alpha$ for the long and short trajectories converge to the same value, the spatio-spectral structures converge as well to end at the energy cutoff. This confirms that the ring-like structures are indeed well explained by a purely spatial transverse and spectral phase, even with numerical apertures of 0.03 . The number and contrast of the rings predicted by each model do not match. It is not overly surprising, since the atomic phase model is very rudimentary: both $q_{\text {eff }}$ and $\alpha$ depend on the intensity, which is a time-varying quantity. They are taken as constant and equal to the SFA value at the peak intensity in the atomic phase model, which is a very rough assumption. We still see a value in using this model because it allows a useful analogy to the Kerr effect and indicates well the trends, but it has limited predictive value. The SFA model on the other hand matches much better the experimentally observed pattern.

Another feature that can be observed in Fig. 4(a) is a lateral asymmetry of the ring patterns. While this is not fully

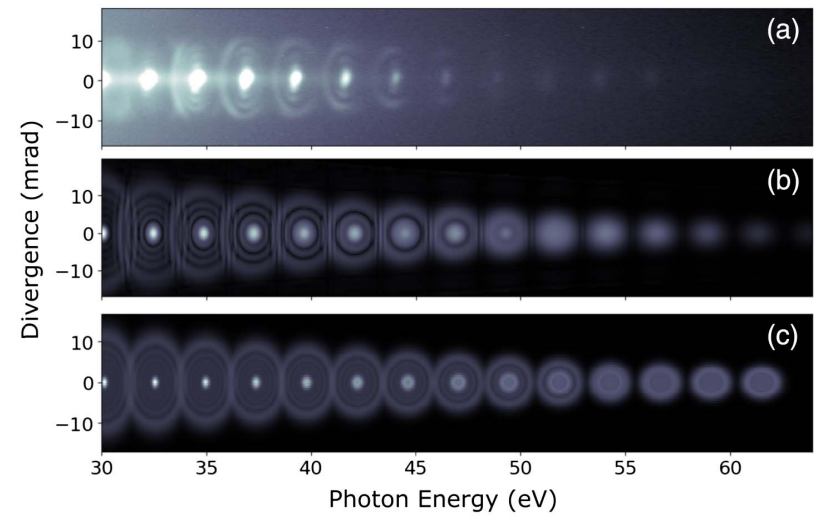

Fig. 4. Spatio-spectral structures obtained with the YDFA in argon. (a) Experiment, (b) SFA model, (c) atomic phase model. 


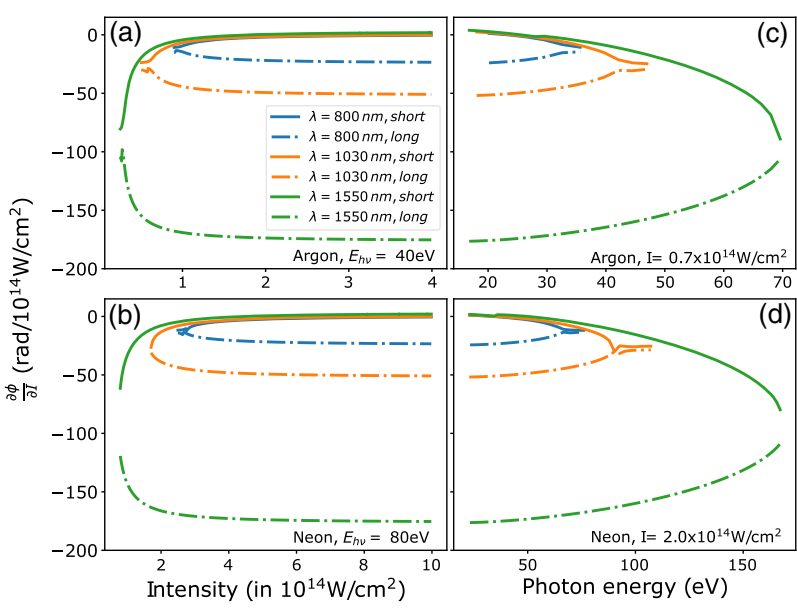

Fig. 5. Atomic phase-intensity coupling constants obtained from the SFA adiabatic model used in the simplified model for three different wavelengths: $800 \mathrm{~nm}$ (blue), $1030 \mathrm{~nm}$ (orange), and $1550 \mathrm{~nm}$ (green) and the two first quantum trajectories labeled short (plain line) and long (dashed line). (Top line) Argon gas. (Bottom line) Neon gas. Left column: variation of $\alpha$ against the laser intensity at a photon energy of (a) $40 \mathrm{eV}$ in argon and (b) $80 \mathrm{eV}$ in neon. Right column: variation of $\alpha$ against the photon energy at an intensity of (c) $0.7 \times 10^{14} \mathrm{~W} / \mathrm{cm}^{2}$ in argon and (d) $2.0 \times 10^{14} \mathrm{~W} / \mathrm{cm}^{2}$ in neon.

understood yet, a possible explanation is related to the temporal profile of the driving pulse: asymmetric pulses will lead to an asymmetry in the XUV spectral broadening. Another source of dissymmetry is the dispersion due to electrons which are being created in the medium as the pulse passes. This changes phasematching conditions but also reshapes the driving pulse. Even with a perfectly symmetric incoming pulse, asymmetric spectral structures might thus appear.

\section{B. Results Obtained with the OPCPA in Argon}

To study the influence of the driving wavelength on these spatio-spectral structures, we now report on experiments performed with the same HHG setup using the OPCPA source at $1.55 \mu \mathrm{m}$. The focus of this part is also to observe the dependence of the spatio-spectral structures on phase-matching conditions; however, the setup is first optimized to obtain the maximum cutoff photon energy. The input beam is focused using a $75 \mathrm{~mm}$ lens in the argon gas jet, resulting in a waist radius of $15 \mu \mathrm{m}$ at the gas target, and an estimated intensity of $1.0 \times 10^{14} \mathrm{~W} / \mathrm{cm}^{2}$. The spectrometer is aligned to observe photon energies from 50 to $100 \mathrm{eV}$.

Figure 6 shows two spatio-spectral structures observed at backing pressures of 7.2 bar and 11.4 bar. A lineout shows the normalized photon flux spectral density obtained, revealing an energy cutoff around $95 \mathrm{eV}$ for both backing pressures. The absolute value of photon flux spectral density obtained at $11.4 \mathrm{bar}$ is also given using the right-hand side vertical scale in Fig. 6(b). As well established in the literature, the gas pressure is an essential parameter that changes longitudinal phasematching conditions by changing the dispersion and absorption of the medium. This is particularly important in tightly focused configurations [27]. A salient feature of the data shown in Fig. 6
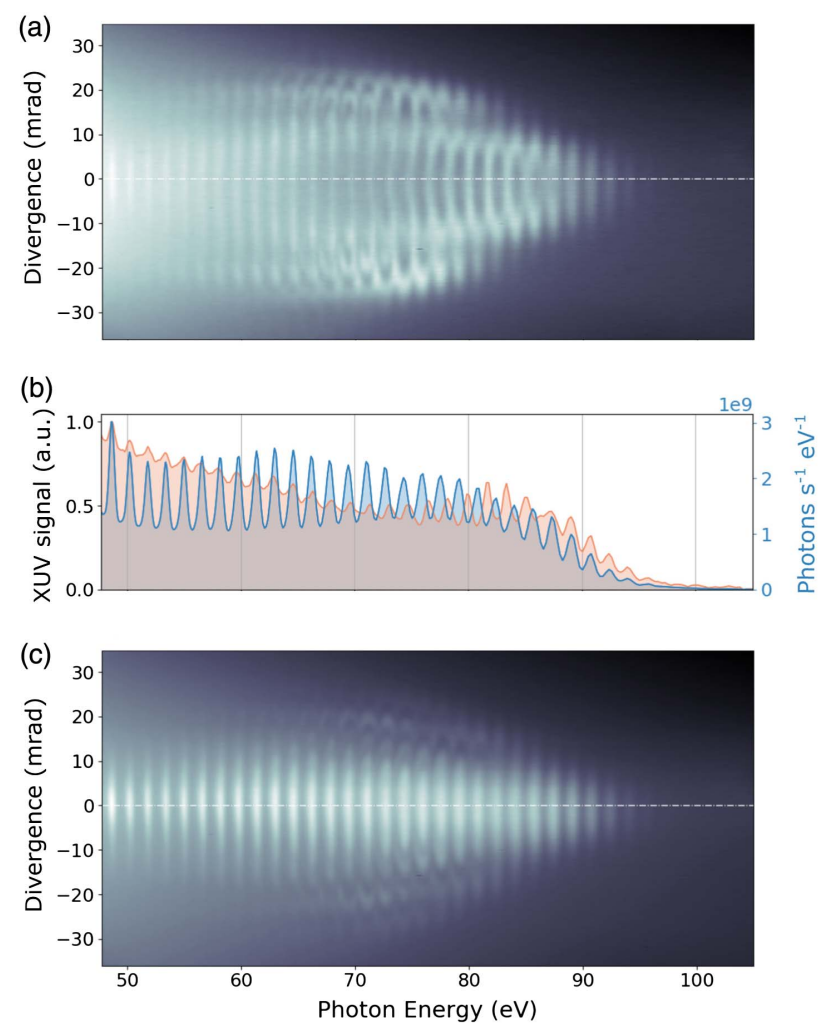

Fig. 6. Experimental spatio-spectral intensity distribution of the XUV beam generated in argon with the OPCPA at (a) 7.2 bar and (c) 11.4 bar. (b) Lineouts of the spectra at 7.2 bar (orange) and 11.4 bar (blue). Both lineouts are normalized on the left axis. In addition, for the blue curve corresponding to panel (c), the right axis indicates the photon flux spectral scale.

is that the change in pressure redistributes the intensity between the more diverging components at angle above $10 \mathrm{mrad}$, associated with the long trajectories, and the central components, associated with the short trajectories, while keeping the underlying structures. This behavior is expected since phase matching is trajectory-specific, while we attribute the geometrical structures to temporal and spatial atomic dipole phase modulation, which is pressure independent.

This allows a direct comparison between the long and short trajectories. The long trajectories observed in Fig. 6(a) follow an increasing divergence as the photon energy decreases from the cutoff value, as already observed in Fig. 4. The ring structure can be distinguished, for example in the region around $70 \mathrm{eV}$ at 10,20 , and $25 \mathrm{mrad}$. The effect of the overlap between the different harmonics increased by the long driving laser wavelength $(1550 \mathrm{~nm})$ is evident in the central lineout of Fig. 6(b). The central ring structure of long trajectories (orange line) is symmetrically located around the short trajectories (blue line) between $75 \mathrm{eV}$ and $82 \mathrm{eV}$ and they overlap for smaller photon energies because the diameter of the ring structures increases. The divergence of the ring structures is more important than in the case of Fig. 4, since the value of alpha is much higher (typically $-150 \times 10^{-14} \mathrm{rad} \cdot \mathrm{cm}^{2} / \mathrm{W}$ for long trajectories and low-order harmonics). As the photon energy increases through the cutoff, the contributions from the short 
trajectories present an increasing divergence [Fig. 6(c)], and long and short trajectories contributions merge at the cutoff.

Since the SFA model gives the contributions of both trajectories, short and long, we used a backing pressure of $6.2 \mathrm{bar}$ that experimentally allows clear observation of both trajectories and compared the result to the SFA model in Fig. 7. Again, the model considers a single plane of Ar atoms located at the input beam waist, thereby neglecting phase-matching effects. The code was run for eight values of the CEP and intensityaveraged, since the experiment was performed with a nonCEP-stabilized laser. The results are in reasonable agreement, and it is clear that the ring features converging at the energy cutoff form the overall arrow shape at the cutoff. The values of divergence are also well reproduced. It is noteworthy that simulation results for a given CEP show interference effects between neighboring spatio-spectral structures, a feature that has been previously observed [28,29].

The ring structures observed in Fig. 7(a) are not perfectly regular, compared to the simulation. Possible experimental causes for irregular ring structures include phase-matching effects that redistribute the intensity among structures, nonperfect temporal or spatial beam profiles of the driving laser leading to asymmetric rings, and slight dissymmetry in the gas expansion, among others.

From an XUV beam user perspective, this experiment shows that using a long-wavelength driver indeed allows us to reach higher energy cutoffs [ $90 \mathrm{eV}$ in argon is not possible with a $800 \mathrm{~nm}$ laser with the same number of cycles $(10$ cycles $\sim 27 \mathrm{fs}$ ) due to the much lower ponderomotive energy], at the cost of increasing divergence due to a large atomic-induced transverse spatial phase.

This strong phase modulation in both the temporal and spatial domains may have far-reaching consequences for potential users. To study the consequence on the temporal attosecond structure, we performed more complete SFA simulations, now taking into account the phase-matching effects. The model, which is described in [30], includes the dispersion and absorption by neutral atoms, electrons, and ions of both
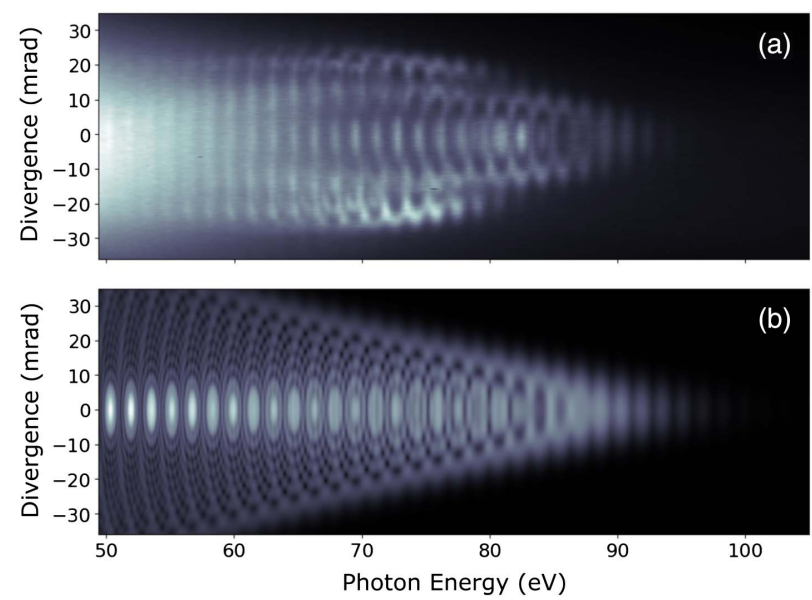

Fig. 7. (a) Experimental spatio-spectral intensity distribution of the XUV beam generated in argon with the OPCPA at 6.2 bar. (b) Result from the SFA simulation code. the fundamental beam and the XUV radiation. The simulation has been done with a $\lambda=1.55 \mu \mathrm{m}$ driving laser, with $50 \mathrm{fs}$ duration, focused down to a $w_{0}=10 \mu \mathrm{m}$ spot size and a peak intensity of $1 \times 10^{14} \mathrm{~W} / \mathrm{cm}^{2}$. The argon gas jet is $300 \mu \mathrm{m}$ long, with a density of $3.75 \times 10^{17}$ atoms $/ \mathrm{cm}^{3}$. The result, considering a single value of CEP, is plotted in Fig. 8. The intensity of HHG radiation is plotted in the time-divergence domain after numerical filtering by a $200 \mathrm{~nm}$ thick aluminum foil and propagation over $1 \mathrm{~m}$. At each angular position, a train of about $1 \mathrm{fs}$ long pulses is obtained. This is consistent with a bandpass of aluminum extending from $15 \mathrm{eV}$ to $70 \mathrm{eV}$ and a regular attochirp [31]. Indeed, a Fourier transformed pulse would yield $\tau_{\mathrm{FT}}=25$ as long pulses. Now, the chirp scales as $\lambda$ and in our conditions, may be estimated as $\phi_{2}=\frac{\partial^{2} \phi}{\partial \omega^{2}} \simeq 1 \times 10^{-32} \mathrm{~s}^{2}$. Plugging this value in the duration of a chirped pulse, $\tau_{\text {out }}=\frac{\sqrt{\tau_{\mathrm{FT}}^{4}+16(\ln 2)^{2} \phi_{2}^{2}}}{\tau_{\mathrm{FT}}}$, gives a duration of $1.1 \mathrm{fs}$, compatible with this numerical observation. The pulse train should thus be compressible using standard methods. In addition, a strong curvature of the wavefront is observed. This is well described by the propagation of a $10 \mu \mathrm{m}$ waist Gaussian beam at a $1 \mathrm{~m}$ distance (legend in Fig. 8). A series of lineouts displayed in Fig. 8(b) evidences the similarity of the intensities once corrected for the spherical phase, with slight shifts in time. The time discretization does not allow us to perform reliable summations of these pulses, as it would appear when focused by, e.g., a toroidal mirror. However, it seems that even though the spectrum is very
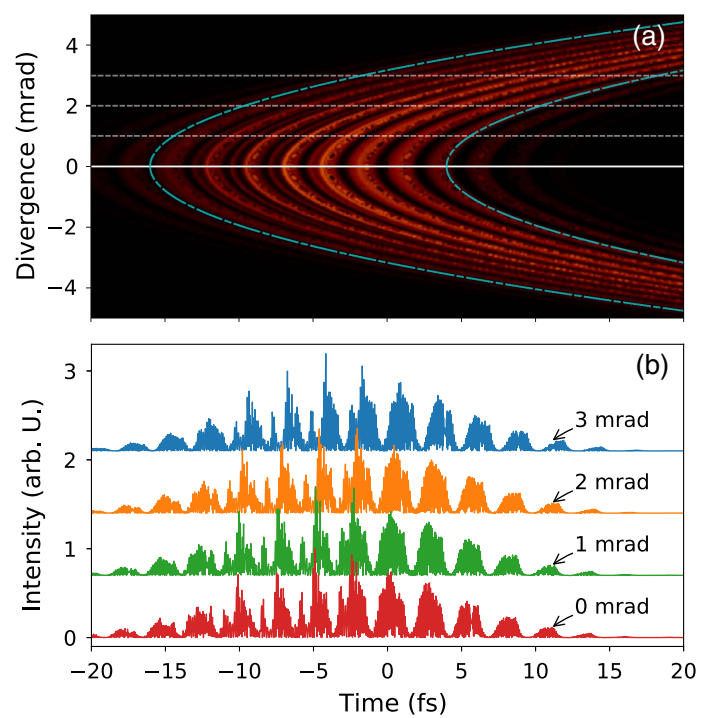

Fig. 8. (a) Spatio-temporal map of the electric field intensity at $z=1 \mathrm{~m}$ from the gas jet, plotted in false colors. The blue dashed lines are two parabolic phase profiles corresponding to a Gaussian beam with $w_{0}=10 \mu \mathrm{m}$ waist at this distance, given by $t+\frac{1}{2 c} \frac{z}{1+\frac{2 R}{2}} \theta^{2}=$ cst, where $\theta$ is the divergence. The white lines are the locations of the lineouts displayed in panel (b). (b) Temporal profiles in the far field at three transverse locations. The lineouts have been shifted horizontally by the time lag corresponding to the trivial Gaussian quadratic phase corresponding to each angle [displayed in blue in panel (a)] and vertically by 0.7 from one another for the sake of clarity. 
structured, after adequate spatial filtering in the far field, the attosecond structure might remain controllable at the focal point of an instrument.

To investigate further the properties of the XUV beam driven by high-power emerging drivers from a user perspective, we now focus on experiments performed in neon.

\section{Results Obtained with the YDFA in Neon}

In particular, we explore the energy cutoff and photon flux scalability, using the YDFA laser temporally compressed to the shortest duration of $16 \mathrm{fs}$ with the current setup. Here the setup is aligned to favor short trajectories and obtain maximum photon flux. The laser pulse ( $70 \mu \mathrm{J}$ energy) is focused in a neon gas jet backed by a pressure of 9 bar using a $75 \mathrm{~mm}$ lens. The spectrometer is aligned to observe photon energies up to $160 \mathrm{eV}$. The measured spatio-spectral intensity distribution is shown in Fig. 9, along with the associated photon flux spectral density.

At this pressure level, it is clear from Fig. 9 that phase matching is optimized for the short trajectories, resulting in a low divergence beam. The overall spatio-spectral structure exhibits low couplings, although a slight increase of the divergence is observed as the photon energy approaches the cutoff energy around $140 \mathrm{eV}$. The photon flux spectral density associated with the 73th harmonic at $58 \mathrm{eV}$ is $1.4 \times 10^{12}$ photons $\cdot \mathrm{s}^{-1} \cdot \mathrm{eV}^{-1}$. This corresponds to an average power in this harmonic of $6 \mu \mathrm{W}$, the largest average power reported to our knowledge in this spectral range $[10,27]$. This highlights the fact that these compact laser sources have a great potential for numerous applications that require large photon flux and/or high repetition rates.
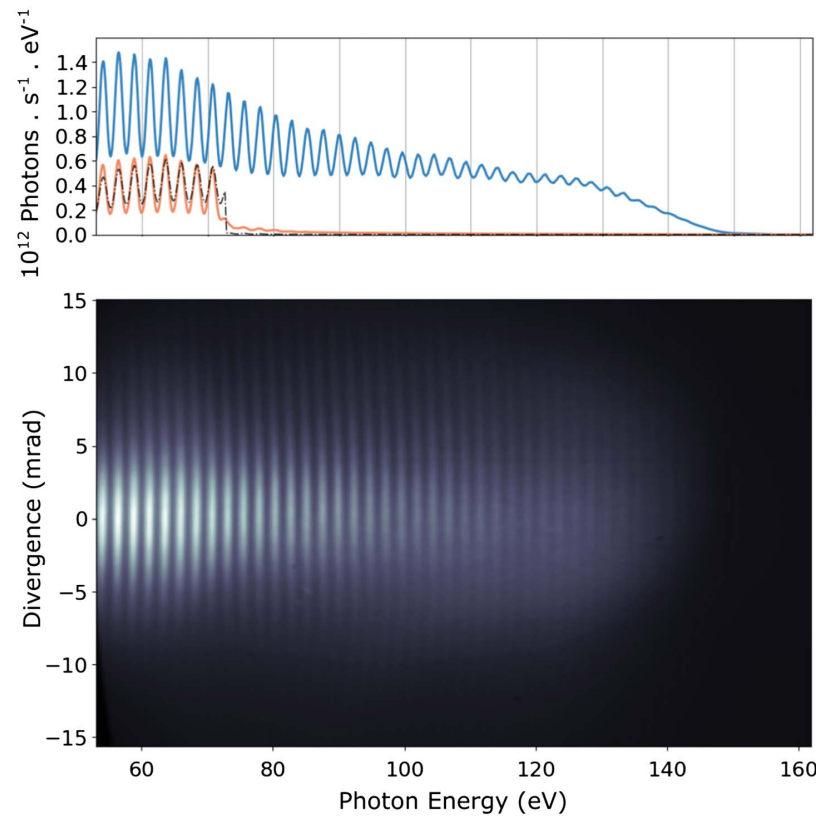

Fig. 9. Bottom: experimental spatio-spectral intensity distribution of the XUV beam generated in neon with the temporally compressed YDFA. Top: measured photon flux spectral density of XUV beam unfiltered (blue) and transmitted through a $200 \mathrm{~nm}$ thick Al filter (orange). Dashed line: measured unfiltered spectrum multiplied by the transmission curve of $200 \mathrm{~nm} \mathrm{Al}$ filter with $20 \mathrm{~nm} \mathrm{Al}_{2} \mathrm{O}_{3}$ layer.

\section{CONCLUSION}

We studied the spatio-spectral intensity distribution of XUV beams generated through HHG using high repetition rate laser systems at $1.03 \mu \mathrm{m}$ and $1.55 \mu \mathrm{m}$ wavelengths. The observed structures are rings at each harmonic with decreasing divergence for increasing photon energies for the long trajectories. For short trajectories, the structures form homogeneous lines with increasing divergence as the photon energy approaches the cutoff. These observations are in line with a simple model that connects these structures to the atomic dipole phase induced in the near field/temporal domain [22]. Since the phase-intensity coupling constant $\alpha$ scales as $\lambda^{3}$, these effects are more prominent as the driver wavelength increases.

At constant peak intensity and pulse duration, the use of a longer-wavelength high repetition rate system results in two effects for the spatio-spectral structures. First, tighter focusing leads to an increased divergence that, in the far field, broadens the ring structure in the spatial dimension, without affecting the photon energy spread. The structure in itself is not modified by the focusing condition, just a scaling is applied. However, in that case, phase matching necessitates higher gas pressure than in loose focusing conditions. Second, the increased driving wavelength leads to a larger spatio-temporal phase modulation that both increases the spatial divergence and photon energy spread and modifies the structure by adding ring patterns. In all cases it is possible to phase match the HHG process to isolate the short trajectories contribution, where these effects are much less pronounced because of the lower value of the phase-intensity coupling constant. Although such an optimization might result in conditions where the overall photon flux is not maximum, this optimization results in usable XUV beams for numerous applications. From this perspective, we have obtained a cutoff energy of $95 \mathrm{eV}$ in argon using the OPCPA driver at $1.55 \mu \mathrm{m}$, and by using the YDFA driver at $1.03 \mu \mathrm{m}$ we achieved a cutoff energy of $140 \mathrm{eV}$ in neon, with photon flux spectral densities up to $3 \times 10^{9}$ photon $\cdot \mathrm{s}^{-1} \cdot \mathrm{eV}^{-1}$ and $1.4 \times 10^{12}$ photon $\cdot \mathrm{s}^{-1} \cdot \mathrm{eV}^{-1}$, respectively. These values are extremely promising for a wide range of applications from gas phase and condensed matter physics.

For instance, the use of coincidence detection devices [32-34] typically requires about $10^{5}-10^{8}$ photons/pulse [32], which matches the number of photons obtained in a single harmonic in the plateau when the short trajectory is phase matched with our sources. This opens the route to the investigation of, e.g., time delays in molecular photoionization [35]. The same applies to solid-state applications, such as, for instance, the attosecond PhotoElectron Emission microscope, which promises the dynamical imaging of localized nanoscale structures, e.g., surface plasmons [36,37]. Also, femtosecond Angularly Resolved PhotoEmission Spectroscopy (FemtoArpes) [38-41] will benefit from these emerging sources with high photon fluxes but low photon number per pulse. Upon delivering the same order of magnitude of photons per pulse, the full Brillouin zone becomes accessible, while keeping a femtosecond to attosecond time resolution [42,43]. It should be noted that for these applications that require a high spectral purity of the XUV beam, the atomic dipole-induced spectral phase has important consequence in terms of each harmonic 
bandwidth. Applications of high repetition rate XUV sources may extend to spin resolved Arpes [44], opening a new route to the study of attosecond and femtosecond dynamics of magnetism. These emerging compact high repetition rate drivers are therefore very promising to broaden the range of applications and spread the use of XUV beams generated through HHG.

Funding. Fonds Unique Interministériel (FUI) (STAR); Conseil départemental de l'Essonne (SOPHIE) (SOPHIE); Agence Nationale de la Recherche (ANR) (ANR-10-LABX0039-PALM, ANR-11-EQPX0005-ATTOLAB， ANR-16CE30-0027-01-HELLIX).

\section{REFERENCES}

1. A. McPherson, G. Gibson, H. Jara, U. Johann, T. S. Luk, I. A. McIntyre, K. Boyer, and C. K. Rhodes, "Studies of multiphoton production of vacuum-ultraviolet radiation in the rare gases," J. Opt. Soc. Am. B 4, 595-601 (1987)

2. M. Ferray, A. L'Huillier, X. F. Li, G. Mainfray, and C. Manus, "Multipleharmonic conversion of $1064 \mathrm{~nm}$ radiation in rare gases," J. Phys. B 21, L31-L35 (1988).

3. P. B. Corkum, "Plasma perspective on strong-field multiphoton ionization," Phys. Rev. Lett. 71, 1994-1997 (1993).

4. P. Balcou, P. Salieres, A. L'Huillier, and M. Lewenstein, "Generalized phase-matching conditions for high harmonics: the role of fieldgradient forces," Phys. Rev. A 55, 3204-3210 (1997).

5. M. Lewenstein, P. Balcou, M. Ivanov, A. L'Huillier, and P. B. Corkum, "Theory of high-order harmonic generation by low-frequency laser fields," Phys. Rev. A 49, 2117-2132 (1994).

6. F. Lépine, M. Y. Ivanov, and M. J. J. Vrakking, "Attosecond molecular dynamics: fact or fiction?" Nat. Photonics 8, 195-204 (2014).

7. M. F. Ciappina, J. A. Pérez-Hernández, A. S. Landsman, W. A. Okell, S. Zherebtsov, B. Förg, J. Schötz, L. Seiffert, T. Fennel, T. Shaaran, T. Zimmermann, A. Chacón, R. Guichard, A. Zaïr, J. W. G. Tisch, J. P. Marangos, T. Witting, A. Braun, S. A. Maier, L. Roso, M. Krüger, P. Hommelhoff, M. F. Kling, F. Krausz, and M. Lewenstein, "Attosecond physics at the nanoscale," Rep. Prog. Phys. 80, 054401 (2017).

8. F. Calegari, G. Sansone, S. Stagira, C. Vozzi, and M. Nisoli, "Advances in attosecond science," J. Phys. B 49, 062001 (2016).

9. E. Lorek, E. W. Larsen, C. M. Heyl, S. Carlström, D. Palecek, D. Zigmantas, and J. Mauritsson, "High-order harmonic generation using a high-repetition-rate turnkey laser," Rev. Sci. Instrum. 85, 123106 (2014).

10. S. Hädrich, J. Rothhardt, M. Krebs, S. Demmler, A. Klenke, A. Tünnermann, and J. Limpert, "Single-pass high harmonic generation at high repetition rate and photon flux," J. Phys. B 49, 172002 (2016).

11. A. Cabasse, G. Machinet, A. Dubrouil, E. Cormier, and E. Constant, "Optimization and phase matching of fiber-laser-driven high-order harmonic generation at high repetition rate," Opt. Lett. 37, 4618-4620 (2012).

12. S. Hädrich, M. Krebs, J. Rothhardt, H. Carstens, S. Demmler, J. Limpert, and A. Tünnermann, "Generation of $\mu \mathrm{W}$ level plateau harmonics at high repetition rate," Opt. Express 19, 19374-19383 (2011).

13. J. Boullet, Y. Zaouter, J. Limpert, S. Petit, Y. Mairesse, B. Fabre, J. Higuet, E. Mével, E. Constant, and E. Cormier, "High-order harmonic generation at a megahertz-level repetition rate directly driven by an ytterbium-doped-fiber chirped-pulse amplification system," Opt. Lett. 34, 1489-1491 (2009).

14. K.-H. Hong, C.-J. Lai, J. P. Siqueira, P. Krogen, J. Moses, C.-L. Chang, G. J. Stein, L. E. Zapata, and F. X. Kärtner, "Multi-mJ, $\mathrm{kHz}, 2.1 \mu \mathrm{m}$ optical parametric chirped-pulse amplifier and high-flux soft x-ray high-harmonic generation," Opt. Lett. 39, 3145-3148 (2014).

15. X. Zhou, P. Ranitovic, C. Hogle, J. Eland, H. Kapteyn, and M. Murnane, "Probing and controlling non-Born-Oppenheimer dynamics in highly excited molecular ions," Nat. Phys. 8, 232-237 (2012).
16. C.-T. Chiang, M. Huth, A. Trützschler, M. Kiel, F. O. Schumann, J. Kirschner, and W. Widdra, "Boosting laboratory photoelectron spectroscopy by megahertz high-order harmonics," New J. Phys. 17, 013035 (2015).

17. L. He, P. Lan, Q. Zhang, C. Zhai, F. Wang, W. Shi, and P. Lu, "Spectrally resolved spatiotemporal features of quantum paths in high-order-harmonic generation," Phys. Rev. A 92, 043403 (2015).

18. A. Dubrouil, O. Hort, F. Catoire, D. Descamps, S. Petit, E. Mével, V. Strelkov, and E. Constant, "Spatio-spectral structures in high-order harmonic beams generated with terawatt 10-fs pulses," Nat. Commun. 5, 4637 (2014).

19. F. Schapper, M. Holler, T. Auguste, A. Zar, M. Weger, P. Salières, L. Gallmann, and U. Keller, "Spatial fingerprint of quantum path interferences in high order harmonic generation," Opt. Express 18, 2987-2994 (2010).

20. S. Carlström, J. Preclíková, E. Lorek, E. W. Larsen, C. M. Heyl, D. Palecek, D. Zigmantas, K. J. Schager, M. B. Gaarde, and J. Mauritsson, "Spatially and spectrally resolved quantum path interference with chirped driving pulses," New J. Phys. 18, 123032 (2016).

21. C. M. Heyl, J. Güdde, U. Höfer, and A. L'Huillier, "Spectrally resolved maker fringes in high-order harmonic generation," Phys. Rev. Lett. 107, 033903 (2011).

22. F. Catoire, A. Ferré, O. Hort, A. Dubrouil, L. Quintard, D. Descamps, S. Petit, F. Burgy, E. Mével, Y. Mairesse, and E. Constant, "Complex structure of spatially resolved high-order-harmonic spectra," Phys. Rev. A 94, 063401 (2016).

23. P. Rigaud, A. Van de Walle, M. Hanna, N. Forget, F. Guichard, Y. Zaouter, K. Guesmi, F. Druon, and P. Georges, "Supercontinuumseeded few-cycle mid-infrared OPCPA system," Opt. Express 24, 26494-26502 (2016).

24. L. Lavenu, M. Natile, F. Guichard, Y. Zaouter, M. Hanna, E. Mottay, and P. Georges, "High-energy few-cycle Yb-doped fiber amplifier source based on a single nonlinear compression stage," Opt. Express 25, 7530-7537 (2017).

25. H. Bandulet, D. Comtois, A. Shiner, C. Trallero-Herrero, N. Kajumba, T. Ozaki, P. Corkum, D. Villeneuve, J. Kieffer, and F. Légaré, "High harmonic generation with a spatially filtered optical parametric amplifier," J. Phys. B 41, 245602 (2008).

26. M. Högner, "HHG max code," https://github.com/Leberwurscht/ HHGmax.

27. C. M. Heyl, C. L. Arnold, A. Couairon, and A. L'Huillier, "Introduction to macroscopic power scaling principles for high-order harmonic generation," J. Phys. B 50, 013001 (2017).

28. E. Mansten, J. M. Dahlstrom, J. Mauritsson, T. Ruchon, A. L'Huillier, J. Tate, M. B. Gaarde, P. Eckle, A. Guandalini, M. Holler, F. Schapper, L. Gallmann, and U. Keller, "Spectral signature of short attosecond pulse trains," Phys. Rev. Lett. 102, 083002 (2009).

29. G. Sansone, E. Benedetti, J.-P. Caumes, S. Stagira, C. Vozzi, M. Pascolini, L. Poletto, P. Villoresi, S. De Silvestri, and M. Nisoli, "Measurement of harmonic phase differences by interference of attosecond light pulses," Phys. Rev. Lett. 94, 193903 (2005).

30. T. Auguste, O. Gobert, T. Ruchon, and F. Quéré, "Attosecond lighthouses in gases: a theoretical and numerical study," Phys. Rev. Lett. A 93, 033825 (2016).

31. S. B. Schoun, R. Chirla, J. Wheeler, C. Roedig, P. Agostini, L. F. DiMauro, K. J. Shafer, and M. B. Gaarde, "Attosecond pulse shaping around a Cooper minimum," Phys. Rev. Lett. 112, 153001 (2014).

32. P. Billaud, M. Géléoc, Y. J. Picard, K. Veyrinas, J. F. Hergott, S. M. Poullain, P. Breger, T. Ruchon, M. Roulliay, F. Delmotte, F. Lepetit, A. Huetz, B. Carré, and D. Dowek, "Molecular frame photoemission in dissociative ionization of $\mathrm{H}_{2}$ and $\mathrm{D}_{2}$ induced by high harmonic generation femtosecond XUV pulses," J. Phys. B 45, 194013 (2012).

33. Y. J. Picard, B. Manschwetus, M. Géléoc, M. Böettcher, E. M. S. Casagrande, N. Lin, T. Ruchon, B. Carré, J.-F. Hergott, F. Lepetit, R. Taëb, A. Maquet, and A. Huetz, "Attosecond evolution of energyand angle-resolved photoemission spectra in two-color (XUV + IR) ionization of rare gases," Phys. Rev. A 89, 031401 (2014).

34. K. Veyrinas, V. Gruson, S. Weber, L. Barreau, T. Ruchon, J.-F. Hergott, J.-C. Houver, R. Lucchese, P. Salières, and D. Dowek, "Molecular frame photoemission by a comb of elliptical high-order 
harmonics: a sensitive probe of both photodynamics and harmonic complete polarization state," Faraday Discuss. 194, 161-183 (2016).

35. P. Hockett, E. Frumker, D. M. Villeneuve, and P. B. Corkum, "Time delay in molecular photoionization," J. Phys. B 49, 095602 (2016).

36. S. H. Chew, K. Pearce, C. Späth, A. Guggenmos, J. Schmidt, F. Süßmann, M. F. Kling, U. Kleineberg, E. Mårsell, C. L. Arnold, E. Lorek, P. Rudawski, C. Guo, M. Miranda, F. Ardana, J. Mauritsson, A. L'Huillier, and A. Mikkelsen, "Imaging Localized Surface Plasmons by Femtosecond to Attosecond Time-Resolved Photoelectron Emission Microscopy-ATTO-PEEM," (Wiley-VCH Verlag GmbH \& Co. KGaA, 2014), pp. 325-364.

37. A. Mikkelsen, J. Schwenke, T. Fordell, G. Luo, K. Klünder, E. Hilner, N. Anttu, A. Zakharov, E. Lundgren, J. Mauritsson, J. N. Andersen, H. Q. Xu, and A. L'huillier, "Photoemission electron microscopy using extreme ultraviolet attosecond pulse trains," Rev. Sci. Instrum. 80, 123703 (2009)

38. S. Eich, A. Stange, A. Carr, J. Urbancic, T. Popmintchev, M. Wiesenmayer, K. Jansen, A. Ruffing, S. Jakobs, T. Rohwer, S. Hellmann, C. Chen, P. Matyba, L. Kipp, K. Rossnagel, M. Bauer, M. Murnane, H. Kapteyn, S. Mathias, and M. Aeschlimann, "Timeand angle-resolved photoemission spectroscopy with optimized high-harmonic pulses using frequency-doubled Ti:sapphire lasers," J. Electron Spectrosc. Relat. Phenom. 195, 231-236 (2014).
39. M. Puppin, Y. Deng, O. Prochnow, J. Ahrens, T. Binhammer, U. Morgner, M. Krenz, M. Wolf, and R. Ernstorfer, "500 kHz OPCPA delivering tunable sub-20 fs pulses with $15 \mathrm{w}$ average power based on an all-ytterbium laser," Opt. Express 23, 1491-1497 (2015).

40. R. Locher, L. Castiglioni, M. Lucchini, M. Greif, L. Gallmann, J. Osterwalder, M. Hengsberger, and U. Keller, "Energy-dependent photoemission delays from noble metal surfaces by attosecond interferometry," Optica 2, 405-410 (2015).

41. G. Rohde, A. Hendel, A. Stange, K. Hanff, L.-P. Oloff, L. X. Yang, K. Rossnagel, and M. Bauer, "Time-resolved ARPES with sub-15 fs temporal and near Fourier-limited spectral resolution," Rev. Sci. Instrum. 87, 103102 (2016).

42. Z. Tao, C. Chen, T. Szilvasi, M. Keller, M. Mavrikakis, H. Kapteyn, and M. Murnane, "Direct time-domain observation of attosecond final-state lifetimes in photoemission from solids," Science 353, 62-67 (2016).

43. M. Lucchini, L. Castiglioni, L. Kasmi, P. Kliuiev, A. Ludwig, M. Greif, J. Osterwalder, M. Hengsberger, L. Gallmann, and U. Keller, "Lightmatter interaction at surfaces in the spatiotemporal limit of macroscopic models," Phys. Rev. Lett. 115, 137401 (2015).

44. C. Nicholson, C. Monney, R. Carley, B. Frietsch, J. Bowlan, M. Weinelt, and M. Wolf, "Ultrafast spin density wave transition in chromium governed by thermalized electron gas," Phys. Rev. Lett. 117, 136801 (2016). 